\title{
Spin-orbit optomechanics of optically levitated chiral Bragg microspheres
}

\author{
Georgiy Tkachenko, Mushegh Rafayelyan, and Etienne Brasselet* \\ Univ. Bordeaux, LOMA, UMR 5798, F-33400 Talence, France \\ and CNRS, LOMA, UMR 5798, F-33400 Talence, France
}

\begin{abstract}
We explore the spin-orbit nature of the optical torque exerted on chiral liquid-crystal microspheres exhibiting circular Bragg reflection. Experimental investigation relies on the direct optomechanical observation of spinning liquid-crystal droplets immersed in water and held in a circularly polarized laser levitator. More generally, we anticipate that the total angular momentum transfer per photon may depart from the commonly assumed spin-only $\pm 2 \hbar$ contribution, when the topological features of the illuminated microsphere are taken into account.
\end{abstract}

\section{INTRODUCTION}

A mechanical consequence of angular momentum transfer from light to matter is the appearance of an optical torque that may actuate the rotational degrees of freedom of an illuminated object. This is widely used to manipulate lightactuated microparticles and micromachines. In practice, the spin and/or orbital contributions to the total optical angular momentum-which respectively refer to polarization and spatial degree of freedom of light-have been exploited via dissipative and nondissipative angular momentum transfer processes [1,2]. Although the mechanical equivalence of spin and orbital angular momentum of light has been experimentally demonstrated $[3,4]$, these two kinds of momenta have distinct features. In particular, the magnitude of spin angular momentum per photon is bounded to $\pm \hbar$, whereas the orbital angular momentum per photon is formally unbounded [5]. Accordingly, the angular momentum transfer associated with the spin torque is bounded to $\pm 2 \hbar$ per photon, in contrast with its orbital counterpart that is associated with arbitrary amount of $\hbar$ angular momentum transfer per photon. Since the optical torque is usually rather weak (indeed, a transfer of $\hbar$ angular momentum per photon to matter produces a modest optical torque of the order of $1 \mathrm{fN} \mathrm{m} \mathrm{W}^{-1}$ ), its optomechanical implementation is generally associated with efforts to optimize angular momentum transfer processes.

Regarding the spin torque, this is usually achieved experimentally using transmission through homogeneous uniaxial anisotropic media providing phase retardation of $\pi$, as Beth did 80 years ago to detect and measure the spin angular momentum of light by using a half-wave plate [6]. On the other hand, the realization of tailor-made structured matter is required in the orbital case; see, for instance, Ref. [7] that reports on orbital angular momentum transfer to turbinelike microrotors up to $\simeq 35 \hbar$ per photon. Noteworthy, it is also possible to combine optimization of both spin and orbital contributions within a single optical process, namely the spin-orbit interaction of light [8]. This particular case refers, for instance, to structured half-wave retarders with in-plane orientation of the optical axis of the form $\psi=q \phi$, where $q$ is a half-integer number and $\phi$ is the polar angle. Indeed, if exposed to a circularly polarized beam with helicity $\Lambda= \pm 1$,

\footnotetext{
*etienne.brasselet@u-bordeaux.fr
}

which carries $\Lambda \hbar$ of spin angular momentum per photon along the propagation direction, such an optical element acquires the net angular momentum $2 \Lambda(1-q) \hbar$ per photon [9]. To date, there is no direct experimental evidence supporting this general prediction, except for several studies dealing with the particular case $q=0$ after the pioneering experiment by Beth. Still, the indirect signature of the $q$ dependence has been obtained via the rotational Doppler shift experiments $[10,11]$. Here we explore the direct optomechanical observation of such a spin-orbit torque by using laser-levitated chiral liquid-crystal droplets that exhibit the circular Bragg reflection phenomenon [12]. More generally, we point out that the total angular momentum transfer per photon may depart from the spin-only $\pm 2 \hbar$ contribution assumed so far $[13,14]$. In the context of applied chiral optomechanics [15-18], this may have implications towards the elaboration of optical enantioseparation techniques, which so far have been exploiting only spin-driven discriminatory optical forces [19-23].

A chiral nematic liquid crystal (or cholesteric) is characterized by a helical supramolecular ordering with handedness $\chi= \pm 1$ (the sign refers to right- and left-handed helices) and pitch $p$ being the distance over which the molecular orientation rotates by $2 \pi$. Cholesterics are well known for the circular Bragg reflection phenomenon, which corresponds to the helicity-preserving resonant reflection of light satisfying the condition $\Lambda \chi=-1$ for a finite spectral range that depends on the angle of incidence with respect to the cholesteric axis [12]. In turn, the circular Bragg reflection is thus characterized by the reversal of the projection of the spin angular momentum along the propagation direction of the incident field. This results in $-2 \chi \hbar$ spin angular momentum transfer per photon, in stark contrast to reflection off a conventional mirror that is associated with no net transfer of angular momentum. So far it is the above spin-only angular momentum balance that has been used to describe the rotational consequences of the circular Bragg reflection on cholesteric droplets $[13,14,18]$. However, liquid crystals may exhibit a rich variety of textures and topological defects [24]. This is especially true for cholesteric droplets, as documented in several experimental studies a few decades ago [25,26] and also in recent ones [27-29] following the resurgence of interest to such structures exhibiting a complex interplay between spatial confinement and chirality. Therefore, revisiting the fundamentals of light-matter interaction for chiral liquid-crystal droplets in the presence of optical angular momentum transfer should contribute to the 

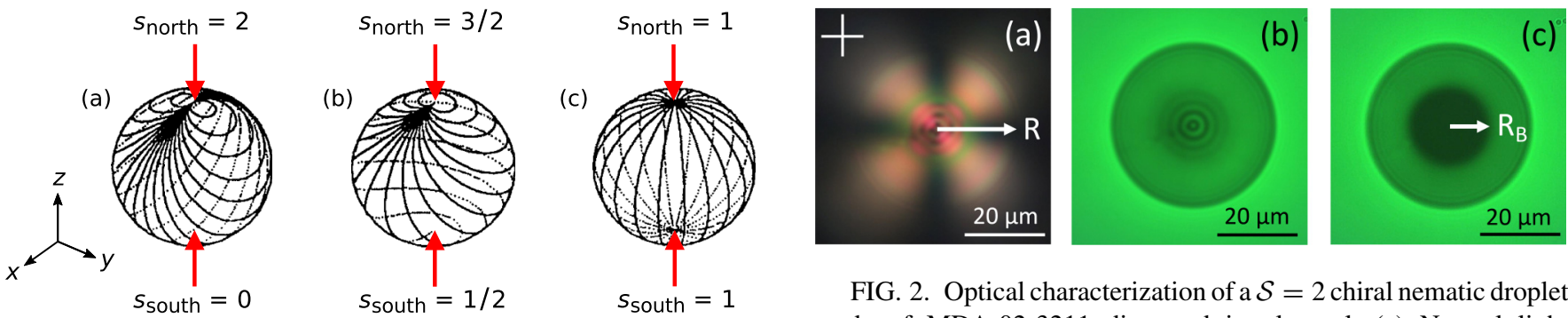

FIG. 2. Optical characterization of a $\mathcal{S}=2$ chiral nematic droplet made of MDA-02-3211 dispersed in glycerol. (a) Natural light imaging between crossed linear polarizers whose orientation is given by the white cross. (b),(c) Full transmission image of the droplet under circularly polarized incoherent illumination at $\lambda=532 \mathrm{~nm}$ with $\Lambda \chi=1$ [no circular Bragg reflection, panel (b)] and $\Lambda \chi=-1$ [circular Bragg reflection, panel (c)]. The central dark disk of radius $R_{\mathrm{B}}$ in panel (c) outlines the area exhibiting circular Bragg reflection. $s_{\text {south }}=2-\mathcal{S}$. (a) $\mathcal{S}=2$. (b) $\mathcal{S}=3 / 2$. (c) $\mathcal{S}=1$. Adapted from Ref. [30].

design of novel chiral architectures and optical manipulation techniques.

\section{CHIRAL LIQUID-CRYSTAL DROPLETS}

In our experiments, we use cholesteric droplets with parallel surface molecular anchoring conditions. Droplets with radius $R \sim 10 \mu \mathrm{m}$ are prepared in pure water by mechanical stirring of the right-handed $(\chi=+1)$ chiral liquid-crystal mixture MDA-02-3211 (from Merck) that is characterized by pitch $p=347 \mathrm{~nm}$ at room temperature. Such droplets may exhibit different kinds of orientational structure, as explored in various experimental $[25,26]$ and theoretical $[30,31]$ studies. The three-dimensional (3D) distribution of the director $\mathbf{n}$ (that is a unit vector representing the local average molecular orientation) for these structures can be described analytically in a few cases, at least to a first approximation. Some of these models are illustrated in Fig. 1 that depicts droplets in the spherical coordinate system $\left(\mathbf{e}_{r}, \mathbf{e}_{\theta}, \mathbf{e}_{\varphi}\right)$ by the ansatz [30] $\mathbf{n}=\cos \Theta(\varphi, r) \mathbf{e}_{\theta}+\sin \Theta(\varphi, r) \mathbf{e}_{\varphi}$, where $\Theta=$ $(\mathcal{S}-1) \varphi+2 \pi r / p+\Theta_{0}$, with $\Theta_{0}$ being a constant, $\mathcal{S}=2$ [Fig. 1(a)], $\mathcal{S}=3 / 2$ [Fig. 1(b)], and $\mathcal{S}=1$ [Fig. 1(c)]. Since $\mathbf{n}$ is everywhere tangential to any spherical surface with radius $r \leqslant R$, topological constraints imply the appearance of point defects with the sum of topological charges always equal to 2 [32]. In the cases shown in Fig. 1, there are either one or two point defects located at the north or south poles of the sphere, with topological charges $s_{\text {north }}=\mathcal{S}$ and $s_{\text {south }}=2-\mathcal{S}$. We note that for $\mathcal{S}=2$ there is no defect at the south pole; still $s_{\text {south }}=0$ is introduced in order to emphasize a locally uniform director pattern.

Our approach relies on the circular Bragg reflection exhibited by a droplet with $\mathcal{S}=2$ (which is the most stable structure when $R \gg p$ [31]) manipulated by a laser beam at wavelength $\lambda=532 \mathrm{~nm}$ which satisfies the circular Bragg reflection condition, namely $n_{\perp} p<\lambda<n_{\|} p$ where $n_{\perp}$ and $n_{\|}$are the refractive indices perpendicular and parallel to the director. Structural identification of such a droplet is performed by means of polarized optical microscopy. Indeed, the dark cross observed in crossed linear polarizer imaging, see Fig. 2(a), confirms the radial distribution of supramolecular helices. The occurrence of circular Bragg reflection is assessed from full transmission imaging under circularly polarized illumination as presented in Figs. 2(b) and 2(c) for $\Lambda \chi= \pm 1$. Due to the blueshift of the photonic band gap associated with circular Bragg reflection at oblique incidence [12], the optical signature of the Bragg reflection off the droplet appears as a dark disk of radius $R_{\mathrm{B}}$ [21]; see Fig. 2(c).

\section{OPTICAL VORTEX LEVITATOR}

In practice we seek an experimental configuration where a $\mathcal{S}=2$ droplet: (i) stays at a fixed location, (ii) is free to spin, (iii) is located far from any solid boundaries, and (iv) preserves the spatial orientation of its radial defect [which lies along the $+z$ axis in Fig. 1(a)].

This configuration is achieved by building a circularly polarized optical vortex levitator sketched in Fig. 3(a). Its operation relies on the use of a moderately focused laser beam carrying an optical vortex with topological charge $\ell= \pm 1$, which refers to an azimuthal dependence of the amplitude proportional to $\exp (i \ell \phi), \phi$ being the polar angle in the
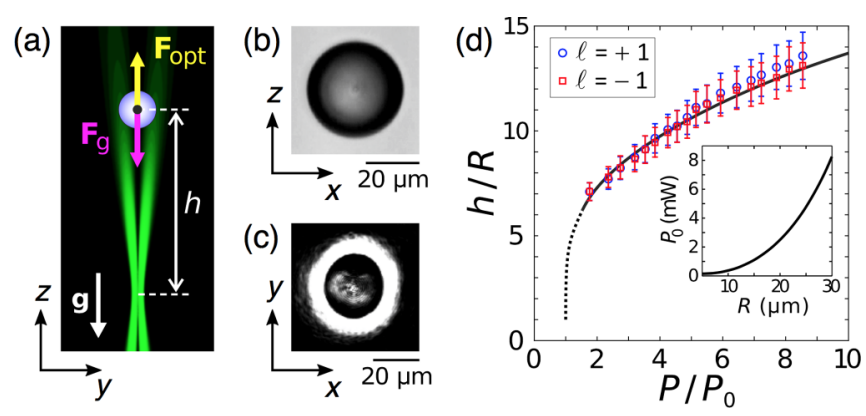

FIG. 3. (a) Sketch of the optical vortex levitation arrangement. Cholesteric droplets are prepared in pure water within a sealed square $\left(1 \mathrm{~mm}^{2}\right)$ glass capillary oriented along the $x$ axis. The beam waist radius of the incident vortex laser beam with topological charge $\ell= \pm 1$ (assuming a Laguerre-Gauss profile) is $w_{0} \simeq 1.5 \mu \mathrm{m}$, the divergence angle is $\theta_{0} \simeq 6.3^{\circ}$, and wavelength is $\lambda=532 \mathrm{~nm}$. Side and top view images of a steadily levitated droplet in water are shown in panels (b) and (c). (d) Normalized droplet elevation vs reduced incident beam power $P / P_{0}$ for a droplet with $R \approx 20 \mu \mathrm{m}$. Curves refer to calculations (see text for details). Inset: calculated minimum power $P_{0}$ required to lift a droplet of radius $R$. 
transverse plane. The vortex beam is directed upwards and satisfies the circular Bragg condition for a droplet immersed in pure water and located around the beam axis $z$, where the incident light intensity is zero. The scattering optical force exerted on the droplet, $\mathbf{F}_{\text {opt }}$, counteracts the gravitational force, $\mathbf{F}_{\mathrm{g}}$. For an incident optical power $P$ exceeding a minimum value $P_{0}$, the two forces are mutually compensated at a distance $h$ above the vortex beam focal plane; see Fig. 3(a). Indeed, the optical force magnitude $F_{\text {opt }}$ decreases with the droplet elevation while $F_{\mathrm{g}}$ is constant. Side view (captured through a red filter) and top view images of a steadily levitated droplet are shown in Figs. 3(b) and 3(c), respectively (see media files 1 and 2).

Normalized droplet elevation $h / R$ vs reduced incident beam power $P / P_{0}$ for a droplet with $R \approx 20 \mu \mathrm{m}$ is measured in the power range $4 \mathrm{~mW}<P<19.5 \mathrm{~mW}$ for $\ell= \pm 1$. Experimental results are shown in Fig. 3(d). The observed elevation and stability of the levitated droplet are numerically analyzed using the ray-optics model presented in Ref. [23] (see Appendix A 1). Simulations have been made using the characteristic internal Bragg angle $\theta_{\mathrm{B}}=21^{\circ}$ that is related to the Bragg radius $R_{\mathrm{B}}$ via the relationship $R_{\mathrm{B}} / R=\left(n / n_{\text {water }}\right) \sin \theta_{\mathrm{B}}$ [21], where $n_{\text {water }}=1.33$ is the refractive index of water and $n=1.6$ is the average value for the liquid crystal. The chosen $\theta_{\mathrm{B}}$ value corresponds to the satisfactory quantitative description of three independent optomechanical experimental studies with the same cholesteric material [21-23]. The model predictions quantitatively agree with experimental data, the only adjustable parameter being a constant elevation offset appearing due to the limited field of view of the side camera. The solid curve in Fig. 3(d) refers to 3D-stable levitation, whereas the dotted curve corresponds to 1D-stable levitation (along $z$ axis only); see Appendix A 2 for stability analysis. In addition, the inset in Fig. 3(d) shows the calculated minimum optical power $P_{0}$ required for lifting a droplet of radius $R$ (see Appendix A 3).

The above conditions (i)-(iii) are thus fulfilled. Regarding the condition (iv), we note the axial symmetry of the beam intensity profile; hence we expect the radial defect of a steadily levitated droplet to point upwards (along the beam axis) as a result of scattering of light on it. Indeed, we confirm this statement by side view observations of the levitating droplet. In other words, the $z$ axes in Figs. 1 and 3(a) match and the levitated droplet is illuminated from the south pole. We note that, in practice, the radial defect remains rather hard to visualize when dealing with helix pitch of a few hundreds of nanometers, as is the case here. However, its experimental identification is nevertheless possible; see, for instance, Fig. 2(d) of Ref. [22].

\section{ROTATIONAL DYNAMICS}

Spinning of a levitated droplet is analyzed via the Braggreflected light, which is registered by a video camera placed after being (ideally) separated from the background light of opposite helicity by means of a polarizing beam splitter and a quarter-wave plate (see Appendix B). Typical intensity patterns for $\ell= \pm 1$ are shown in Figs. 4(a) and 4(c), which correspond to the virtual focal plane of the droplet acting as a convex spherical mirror imaged by the levitator microscope
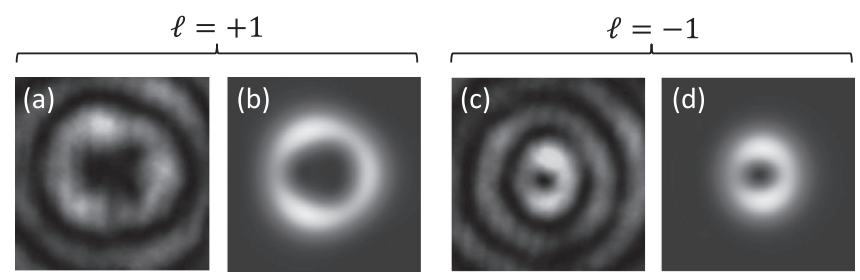

FIG. 4. Experimental (a),(c) and calculated (b),(d) intensity profiles of the virtual focal plane of the Bragg reflecting droplet for $\ell=+1$ (a),(b) and $\ell=-1$ (c),(d). Calculated patterns refer to the formula given in the text with $(\alpha, \beta, \gamma)=(1,0.1,-0.03)$.

objective. Remarkably, the observed doughnutlike intensity profiles demonstrate broken rotational symmetry. On the one hand, the $\ell$-dependent annular features are reminiscent of the spin-orbit interaction arising from the droplet topology, as recently suggested in Ref. [33] which demonstrated that Bragg reflection off the south pole of a $\mathcal{S}=2$ droplet results in light fields appearing as if structured by an effective half-wave retarder with $q_{\text {eff }}=-1$. This imparts to the amplitude of the incident vortex of charge $\ell$ an extra term of the form $\exp \left(2 i \Lambda q_{\text {eff }} \phi\right)$, with $\Lambda q_{\text {eff }}=1$ in our case. On the other hand, here we point out that the broken axisymmetry can be explained from the interference between the expected signal and unavoidable background contribution from the setup. The two latter statements are qualitatively supported by Figs. 4(b) and $4(d)$ that display interference intensity patterns of the form $\left|E_{\text {signal }}+E_{\text {background }}\right|^{2}$, taking generic fields $E_{\text {signal }}=$ $\alpha L G_{0, \ell+2}$ and $E_{\text {background }}=\beta L G_{0, \ell}+\gamma$, where $L G_{0, \ell}(r, \phi)=$ $(r / w)^{|\ell|} e^{-r^{2} / w^{2}} e^{i \ell \phi}$ refers to Laguerre-Gauss modes of radial index zero and azimuthal index $\ell$, and $(\alpha, \beta, \gamma)$ are constants, while a more rigorous description should account for nonparaxial diffraction explaining the observed rings structure, though being beyond the scope of the present work.

In practice, the rotation of droplets is assessed from dynamics of the reflected intensity patterns, see media files 3 and 4 for the videos recorded for $\ell= \pm 1$, which corresponds to Figs. 4(a) and 4(c). The dynamics of the 2D correlation coefficient for the acquired images is analyzed via the power spectra. The sample results for $\ell= \pm 1$ are presented in Fig. 5(a). Noteworthy is that these observations show that the optical torque does not depend on the sign of the topological charge of the incident vortex beam. Namely, the measured peak frequencies $F_{ \pm}$for $\ell= \pm 1$ satisfy $F_{+} / F_{-} \simeq 1$. Given a timeindependent background field, the observed dynamics implies a time-dependent signal. Indeed, the Bragg-reflected light from the spinning droplet experiences a so-called rotational Doppler angular frequency shift $N \Omega$, where $N$ is the amount of total (spin+orbital) angular momentum transferred by light to matter per photon in $\hbar$ units [34] and $\Omega$ is the droplet angular frequency. Therefore $F$ is related to the droplet frequency $f$ via the relationship $F=N f$ and the time-dependent interference pattern is simulated taking $\alpha=e^{i N \Omega t}$; see media files 5 and 6 for $\ell= \pm 1$.

Since we observe a change of both the spin and orbital state for the Bragg-reflected light, our experiments make a direct demonstration of spin-orbit angular optomechanics. However, the definition of spin and orbital contributions to $N$ 

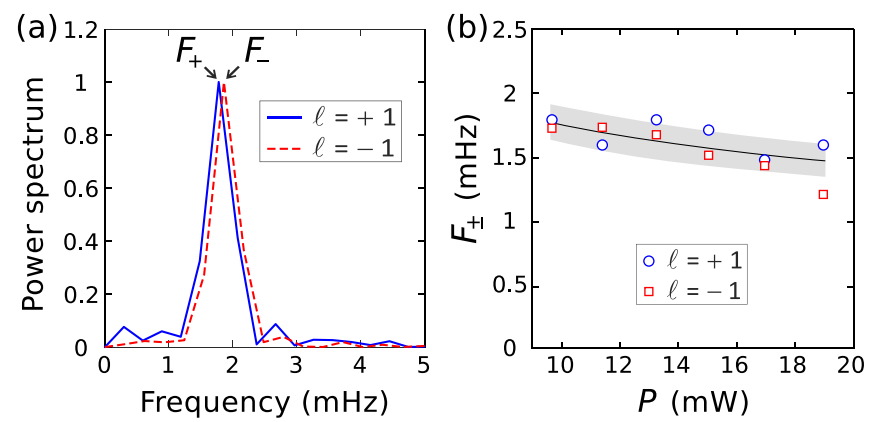

FIG. 5. Analysis of the intensity patterns dynamics for $\ell= \pm 1$. (a) Examples of normalized power Fourier spectra of the autocorrelation dynamics for $R=18.3 \mu$ m levitated at $P=11.4 \mathrm{~mW}$. (b) Measured (markers) and simulated (curve) autocorrelation frequency $F_{ \pm}=N f_{ \pm}$. The simulated curve refers to the best fit that corresponds to $N \hbar$ total angular momentum transfer per photon, $N=1.90 \pm 0.07$. The gray area indicates the standard deviation range of the experimental data.

remains a nontrivial task recalling the complexity of the droplet as a 3D inhomogeneous, anisotropic, nonparaxial and chiral system. Still, within our simplified model, we can evaluate an effective value of $N$. On the one hand, we assume that the incident light impinging on the droplet at distance $r>R_{\mathrm{B}}$ (from the beam axis $z$ ) interacts with the droplet as if it was an isotropic achiral dielectric sphere. This part of the incident light does not contribute to the net optical torque exerted on the droplet. On the other hand, the totally reflected light that corresponds to $r<R_{\mathrm{B}}$ leads to an optical torque with magnitude $\Gamma_{\text {opt }}=N P_{\text {reflected }} / \omega$, where $\omega$ is the angular frequency of light and $P_{\text {reflected }}$ is the optical power intercepted over the circular area of radius $R_{\mathrm{B}}$ (see Appendix A 4). In the limit of small Reynolds number, as is the case in our study, the optical torque is balanced by the drag torque $\Gamma_{\text {visc }}=8 \pi \eta R^{3} \Omega$ [35], where $\eta$ is the dynamical viscosity of the host fluid, taken as $1 \mathrm{mPa}$ s for water at the room temperature. One thus gets $F=N^{2} P_{\text {reflected }} /\left(16 \pi^{2} \eta \omega R^{3}\right)$ and the measured power dependence can be fitted taking $N$ as the only adjustable parameter, which gives $N=1.90 \pm 0.07$; see solid curve in Fig. 5(b). The counterintuitive decrease of the light-induced rotation frequency vs incident power is related to the increase of the levitation altitude $h$ with $P$; see Fig. 3(d).

In addition, the spin-orbit nature of the optical torque in the case of a droplet with $\mathcal{S}=1$ can be grasped from symmetry considerations. Indeed, the invariance per rotation of the structure of the droplet around the pole axis, see Fig. 1(c), implies zero net optical torque; hence $N=0$. In that case, the spin and orbital contribution cancel each other, which appears as the chiral reflective analog of the nonchiral transmissive case of radial nematic droplets trapped in circularly polarized optical tweezers [36].

These two cholesteric examples with $\mathcal{S}=1$ and $\mathcal{S}=2$ satisfy the relationship $N=2 \Lambda\left(1-s_{\text {pole }}\right)$ and we speculate its generalization to any kind of Bragg cholesteric droplet, hence following the general case of transmissive 2D structured half-wave retarders [10]. Consequently, we expect that droplets with $\mathcal{S}=3 / 2$ give $N=-\Lambda$ when illuminated from the north pole, and $N=+\Lambda$ when illuminated from the south pole. Such an experimental demonstration would make a strong support to the predicted angular dynamics caused by spin-orbit interactions between chiral light and chiral Bragg microspheres.

However, in the particular case of $\mathcal{S}=2$ droplets discussed here, we stress that both the proposed interpretation for the observed intensity patterns from Ref. [33] and the evaluation of the effective total angular mometum per active photon from a simplified modeling of a complex spinorbit optical element are seemingly contradicting each other. Namely, topological beam shaping appears dictated by an effective structured half-wave retarder with $q_{\text {eff }}=s_{\text {pole }}-1$, while the net angular momentum transfer is formally governed by $q_{\text {eff }}=s_{\text {pole }}$, which fosters the need for further work.

\section{CONCLUSION}

The effect reported in this study is not restricted to wavelengths inside the circular photonic band gap. Indeed, the physical process at work relies on helicity-preserving reflection, which occurs for arbitrary wavelength when the condition $\Lambda \chi=-1$ is satisfied (although the reflectance drops outside the band gap), while no net torque has been observed so far for the orthogonal circular polarization state [13]. A signature of such wavelength-independent topological beam shaping of helicity-preserved reflected light can be found in a recent experimental work on space-variant chiral Bragg mirrors [37]. From a technical point of view, the proposed strategy to achieve rotational manipulation of chiral nematic droplets in an optical vortex levitator allows exploration of a wide range of droplet radius and overall Bragg optical reflectance, while preserving the $3 \mathrm{D}$ stability of the droplets location, in contrast to previous experimental works so far limited to small radius range and partial reflectance $[13,14]$. More generally, this technique could be applied to other kinds of reflective microparticles and also to absorbing ones via the photophoretic phenomenon. Our results also contribute to emerging investigations of spin-orbit optomechanics under paraxial light fields [10,11]. More generally, the orbital analog of the (spin) circular Bragg reflection is another direction that would be interesting to explore [38].

\section{APPENDIX A: VORTEX LEVITATOR AT WORK}

\section{Optical levitation equilibrium}

In order to simulate radiation forces exerted by a moderately focused optical vortex beam on the Bragg-reflecting cholesteric droplet, we apply the ray optics approach that has already proven its relevance in previous studies [21-23]. The droplet is considered as a uniform dielectric sphere which acquires linear momentum from light by reflection, refraction, and scattering of the incident rays. First, we introduce the spherical coordinate system $(r, \theta, \phi)$ as shown in Fig. 6 and express the elementary optical force exerted on the surface element $d S(\theta, \phi)=R^{2} \sin \theta d \theta d \phi$. As the divergent vortex beam is symmetrical with respect to the axis $z$, it is convenient to express the light field at every 


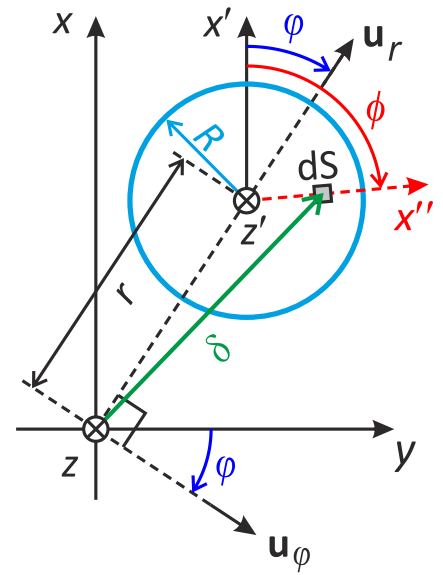

FIG. 6. Definitions and notations for simulation of radiation forces acting on a Bragg-reflecting sphere within a divergent optical vortex beam.

surface element as a function of two characteristic distances, $\xi$ the distance from the $d S$ to the focal plane and $\delta$ the distance from $d S$ to the $z$ axis (see Fig. 6). More precisely, $\xi=-R \cos \theta-\operatorname{sgn}(\cos \theta) z$, with sgn the sign function, and $\delta=\left[r^{2}+2 r R \sin \theta \cos (\phi-\varphi)+R^{2} \sin ^{2} \theta\right]^{1 / 2}$. Assuming the incident vortex beam to be described by a Laguerre-Gauss mode of radial index zero and azimuthal index $\ell$, the intensity profile of the levitator beam is expressed as

$$
I(\delta, \xi)=\frac{2^{|\ell|+1} P}{\pi|\ell| ! w(\xi)^{2}}\left(\frac{\delta}{w(\xi)}\right)^{2|\ell|} \exp \left(-\frac{2 \delta^{2}}{w(\xi)^{2}}\right)
$$

where $w(\xi)=w_{0} \sqrt{1+\left(\xi / z_{0}\right)^{2}}$ and $z_{0}=\pi w_{0}^{2} / \lambda$ is the Rayleigh range. Following Ref. [23], one can show that the net force equals $\mathbf{F}_{\text {opt }}=F_{r}(r, z) \mathbf{u}_{r}+F_{z}(r, z) \mathbf{u}_{z}$ with the radial and axial components

$$
\begin{aligned}
F_{r}(r, z)= & -\frac{n_{\mathrm{ext}} R^{2}}{2 \mathrm{c}} \int_{0}^{2 \pi} \int_{0}^{\pi} I(\delta, \xi) \\
& \times f_{\perp}(\theta) \sin 2 \theta \cos (\phi-\varphi) d \theta d \phi, \\
F_{z}(r, z)= & -\frac{n_{\mathrm{ext}} R^{2}}{2 \mathrm{c}} \int_{0}^{2 \pi} \int_{0}^{\pi} I(\delta, \xi) f_{\|}(\theta) \sin 2 \theta d \theta d \phi,
\end{aligned}
$$

where $n_{\text {ext }}$ is the refractive index of the host fluid (in our case, $n_{\text {ext }}=n_{\text {water }}$ ), c is the speed of light in vacuum, and $f_{\perp}(\theta)$ and $f_{\|}(\theta)$ respectively describe the transverse and longitudinal (relative to the incident ray) changes of the photon linear momentum as a result of interaction with the sphere.

For non-Bragg rays (i.e., rays that impinge onto the droplet at a distance larger than $R_{\mathrm{B}}$ from the droplet axis $z^{\prime}$ ), we get

$$
\begin{gathered}
f_{\perp}(\theta)=\mathcal{R} \sin 2 \theta-\mathcal{T}^{2} \frac{\sin \left(2 \theta-2 \theta_{\text {int }}\right)+\mathcal{R} \sin 2 \theta}{1+2 \mathcal{R} \cos 2 \theta_{\text {int }}+\mathcal{R}^{2}}, \\
f_{\|}(\theta)=1+\mathcal{R} \cos 2 \theta-\mathcal{T}^{2} \frac{\cos \left(2 \theta-2 \theta_{\text {int }}\right)+\mathcal{R} \cos 2 \theta}{1+2 \mathcal{R} \cos 2 \theta_{\text {int }}+\mathcal{R}^{2}},
\end{gathered}
$$

where $\mathcal{R}$ and $\mathcal{T}=1-\mathcal{R}$ are the reflectance and transmittance of the droplet interface, with $\theta_{\text {int }}=$ $\operatorname{sgn}(\cos \theta) \arcsin \left[\left(n_{\text {ext }} / n\right) \sin \theta\right]$ the signed angle of refraction. Accounting for the incident circular polarization state and discarding the polarization projection on the local frame of incidence, we also assume that $\mathcal{R}(\theta)=\left[\mathcal{R}_{\|}(\theta)+\mathcal{R}_{\perp}(\theta)\right] / 2$, with $\mathcal{R}_{\|}(\theta)=\left[\tan \left(\theta-\theta_{\text {int }}\right) / \tan \left(\theta+\theta_{\text {int }}\right)\right]^{2}$ and $\mathcal{R}_{\perp}(\theta)=[\sin (\theta-$ $\left.\left.\theta_{\text {int }}\right) / \sin \left(\theta+\theta_{\text {int }}\right)\right]^{2}$ being the reflectance coefficients of plane waves polarized parallel and perpendicular to the incidence plane. For Bragg rays (i.e., that impinge onto the droplet at a distance smaller than $R_{\mathrm{B}}$ from the droplet axis), we get

$$
\begin{gathered}
f_{\perp}(\theta)=\sin 2 \theta, \\
f_{\|}(\theta)=1+\cos 2 \theta .
\end{gathered}
$$

Given the axial symmetry of the light field, the radial force $F_{r}(r, z)$ becomes zero on the beam axis; hence droplet levitation occurs at $(r, z)=(0, h)$ when the net optical force $\mathbf{F}_{\text {opt }}=F_{z}(r, h) \mathbf{u}_{z}$ balances the gravitational force $\mathbf{F}_{\mathrm{g}}=m \mathbf{g}=$ $-\left(4 / 3 \pi R^{3} \Delta \rho \mathrm{g}\right) \mathbf{u}_{z}$, where $m$ is the droplet mass and $\Delta \rho \simeq$ $65 \mathrm{~kg} \mathrm{~m}^{-3}$ is the difference between the density of the liquid crystal and that of water. Namely,

$$
F_{z}(0, h)-4 / 3 \pi R^{3} \Delta \rho \mathrm{g}=0 .
$$

\section{Optical levitation stability}

However, the equilibrium condition given by Eq. (A8) alone does not guarantee the stability of the optical levitation. Indeed, levitation at $(0, h)$ is stable in $3 \mathrm{D}$ only if small shifts of the droplet from this point result in the net restoring force, i.e., when the following conditions are fulfilled:

$$
\begin{aligned}
& \frac{\partial F_{z}(0, h)}{\partial z}<0, \\
& \frac{\partial F_{r}(0, h)}{\partial r}<0 .
\end{aligned}
$$

Such an analysis allows us to ascertain the stability of the equilibrium position, as shown in Fig. 3(d). In fact, the simulated curve has two branches that are symmetric with respect to $h / R=1$, of which the lower one (not shown) corresponds to unstable equilibrium.

\section{Optical levitation threshold}

As shown in the inset in Fig. 4(d), there is a threshold optical power $P_{0}$ for lifting a droplet of radius $R$. For $P<P_{0}$, the net optical radiation force is too weak for compensating the gravity and the droplet cannot be lifted. This parameter is valuable from the practical point of view as it gives an order of magnitude of the minimum power required for levitating a 
droplet. Our numerical calculations show that threshold optical power $P_{0}$ increases nonlinearly with the droplet radius $R$, as shown in the inset of Fig. 4(d).

Interestingly, the parameter $P_{0}$ can be readily estimated if one takes into account the fact that the beam spot at the focal plane is much smaller than the Bragg-reflecting area of the droplet, i.e., $w_{0}^{2} \ll\left(R \sin \theta_{\mathrm{B}, \mathrm{ext}}\right)^{2}$, which is true for the typical radii $R$ available in the experiment. In this case one can assume that all the incident optical power $P$ is reflected back by the droplet, which consequently gains the linear momentum of $2 \hbar \mathbf{k}$ from every incident photon. By definition, the resulting net optical force exerted on the droplet, $\left(2 P n_{\text {ext }} / \mathrm{c}\right) \mathbf{u}_{z}$, balances the gravitational force $\mathbf{F}_{\mathrm{g}}$ when $P=P_{0}$. Hence the threshold optical power expresses as

$$
P_{0}=\frac{2 \pi \operatorname{cg} \Delta \rho}{3 n_{\mathrm{ext}}} R^{3}
$$

\section{Bragg-reflected optical power}

The optical power $P_{\text {reflected }}$ that corresponds to the reflected power off the Bragg area of radius $R_{\mathrm{B}}$ is evaluated accounting for the intensity distribution of the incident vortex beam at the droplet surface facing the incident beam. Since $\delta=0$ when the droplet is levitated in a stable manner, one gets from Eq. (A1)

$$
P_{\text {reflected }}=\frac{2^{|\ell|+2} P}{|\ell| !} \int_{0}^{R_{\mathrm{B}}} \frac{r^{2|\ell|+1}}{w(\zeta)^{2|\ell|+2}} e^{-\frac{2 r^{2}}{w^{2}(\zeta)}} d r,
$$

where $\zeta=h-R \cos [\arcsin (r / R)]$.

\section{APPENDIX B: EXPERIMENTAL SETUP}

The experimental setup used in the present study is sketched in Fig. 7(a), where $(x, y, z)$ is a right-handed Cartesian coordinate system. The incident vortex beam is prepared as follows. A circularly polarized collimated Gaussian beam from a continuous-wave laser operating at $\lambda=532 \mathrm{~nm}$ propagates along $z$ axis through a space-variant birefringent optical vortex generator from Altechna R\&D. The transmitted beam is contracircularly polarized with respect to the incident one and exhibits a doughnut-shaped transverse intensity profile; see Fig. 7(b). Although such a vortex beam is not a pure transverse mode, its intensity distribution can be closely described by the Laquerre-Gaussian function $\mathrm{LG}_{0 \ell}$ [23]. The sign of $\ell$ depends on the input circular polarization state defined by a quarter-wave plate placed before the vortex generator. A second quarter-wave plate is placed after the vortex generator and makes the vortex beam $p$ polarized in order to allow maximum of light passing through the nonpolarizing and polarizing beam splitters (NPBS and PBS on Fig. 7), after which another quarter-wave plate (QWP on Fig. 7) sets the photon helicity $\Lambda$ for the optical manipulation experiment.

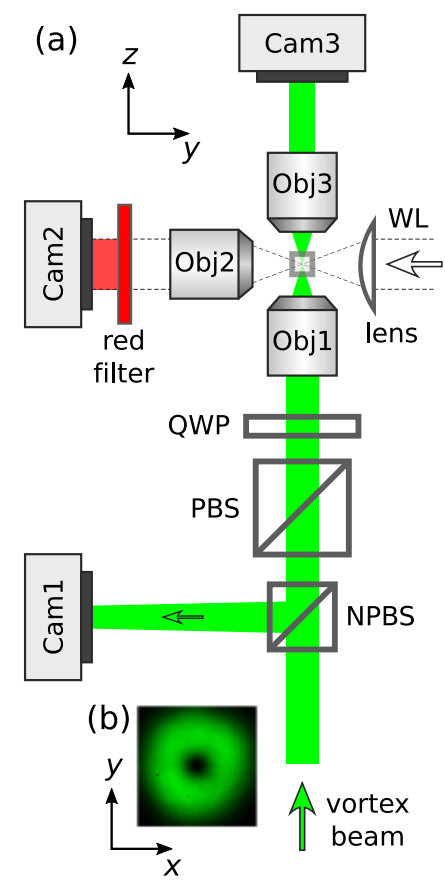

FIG. 7. Sketch of the experimental setup. (a) Optical set-up, not to scale. QWP: quarter-wave plates; NPBS and PBS: nonpolarizing and polarizing beam splitter cubes; Obj1-3: microscope objectives; Cam1-3: CMOS cameras; WL: white light. Measured transverse intensity distributions of the generated vortex beam.

The microscope objective Obj1 (magnification $\times 20$; numerical aperture 0.4 ) with underfilled back aperture is focusing the beam into "funnel" of light with the divergence angle $\theta_{0}$. The experimental sample is a glass capillary $\left(1 \times 1 \mathrm{~mm}^{2}\right.$ square cross section) filled with pure water containing several radial droplets of MDA-02-3211 cholesteric mixture. Using the finding of our previous studies, we consider the cholesteric liquid crystal reflecting $100 \%$ of light with wavelength $532 \mathrm{~nm}$ and helicity $\Lambda=-1$ impinging at the incidence angle between $\left(\pi-\theta_{\mathrm{B}, \text { ext }}\right)$ and $\pi$ (counted off the axis $z$, with $\theta_{\mathrm{B}, \mathrm{ext}} \approx 25^{\circ}$ being the external "Bragg cone" angle that is related to the internal Bragg cone angle $\theta_{\mathrm{B}}$ via $n_{\mathrm{ext}} \sin \theta_{\mathrm{B}, \mathrm{ext}}=n \sin \theta_{\mathrm{B}}$ [21-23]. Side-view and top-view images of the droplet are respectively obtained by the microscope objectives Obj2 and Obj3 (same as Obj1) and recorded by the cameras Cam1 and Cam2. Red filter blocks the green laser illumination and allows side-view imaging of the droplet under red-filtered natural white light (WL); see Fig. 3(b). The laser light reflected off the droplet is collected by Obj1 and propagates back through the retarder QWP and the cube PBS, which transmits only the Bragg-reflected part (which is helicity preserved). The passed light is redirected by the cube NPBS to camera Cam1. The distance from Obj1 to Cam1 is adjusted in order to image the virtual focal plane of the droplet that acts as a convex spherical mirror.
[1] D. G. Grier, A revolution in optical manipulation, Nature (London) 424, 810 (2003).
[2] M. Padgett and R. Bowman, Tweezers with a twist, Nat. Photon. 5, 343 (2011). 
[3] M. E. J. Friese, J. Enger, H. Rubinsztein-Dunlop, and N. R. Heckenberg, Optical angular-momentum transfer to trapped absorbing particles, Phys. Rev. A 54, 1593 (1996).

[4] N. B. Simpson, K. Dholakia, L. Allen, and M. J. Padgett, Mechanical equivalence of spin and orbital angular momentum of light: an optical spanner, Opt. Lett. 22, 52 (1997).

[5] L. Allen, M. W. Beijersbergen, R. J. C. Spreeuw, and J. P. Woerdman, Orbital angular momentum of light and the transformation of Laguerre-Gaussian laser modes, Phys. Rev. A 45, 8185 (1992).

[6] R. A. Beth, Mechanical detection and measurement of the angular momentum of light, Phys. Rev. 50, 115 (1936).

[7] X.-F. Lin, G.-Q. Hu, Q.-D. Chen, L.-G. Niu, Q.-S. Li, A. Ostendorf, and H.-B. Sun, A light-driven turbine-like microrotor and study on its light-to-mechanical power conversion efficiency, Appl. Phys. Lett. 101, 113901 (2012).

[8] K. Y. Bliokh, F. J. Rodríguez-Fortuño, F. Nori, and A. V. Zayats, Spin-orbit interactions of light, Nat. Photon. 9, 796 (2015).

[9] L. Marrucci, C. Manzo, and D. Paparo, Optical Spin-to-Orbital Angular Momentum Conversion in Inhomogeneous Anisotropic Media, Phys. Rev. Lett. 96, 163905 (2006).

[10] D. Hakobyan and E. Brasselet, Left-handed optical radiation torque, Nat. Photon. 8, 610 (2014).

[11] D. Hakobyan and E. Brasselet, Optical torque reversal and spin-orbit rotational doppler shift experiments, Opt. Express 23, 31230 (2015).

[12] M. Faryad and A. Lakhtakia, The circular Bragg phenomenon, Adv. Opt. Photon. 6, 225 (2014).

[13] M. Donato, J. Hernandez, A. Mazzulla, C. Provenzano, R. Saija, R. Sayed, S. Vasi, A. Magazzu, P. Pagliusi, R. Bartolino, P. Gucciardi, O. Marago, and G. Cipparrone, Polarizationdependent optomechanics mediated by chiral microresonators, Nat. Commun. 5, 3656 (2014).

[14] M. G. Donato, A. Mazzulla, P. Pagliusi, A. Magazzu, R. J. Hernandez, C. Provenzano, P. G. Gucciardi, O. M. Marago, and G. Cipparrone, Light-induced rotations of chiral birefringent microparticles in optical tweezers, Sci. Rep. 6, 31977 (2016).

[15] H. F. Gleeson, T. A. Wood, and M. Dickinson, Laser manipulation in liquid crystals: an approach to microfluidics and micromachines, Philos. Trans. R. Soc. London A 364, 2789 (2006).

[16] Y. Yang, P. D. Brimicombe, N. W. Roberts, M. R. Dickinson, M. Osipov, and H. F. Gleeson, Continuously rotating chiral liquid crystal droplets in a linearly polarized laser trap, Opt. Express 16, 6877 (2008).

[17] M. Mosallaeipour, Y. Hatwalne, N. V. Madhusudana, and S. Ananthamurthy, Laser induced rotation of trapped chiral and achiral nematic droplets, J. Mod. Opt. 57, 395 (2010).

[18] R. J. Hernandez, C. Provenzano, A. Mazzulla, P. Pagliusi, M. Viola, and G. Cipparrone, Cholesteric solid spherical microparticles: chiral optomechanics and microphotonics, Liq. Cryst. Rev. 4, 59 (2016).

[19] G. Cipparrone, A. Mazzulla, A. Pane, R. J. Hernandez, and R. Bartolino, Chiral self-assembled solid microspheres: A novel multifunctional microphotonic device, Adv. Mater. 23, 5773 (2011).

[20] R. J. Hernandez, A. Mazzulla, A. Pane, K. Volke-Sepulveda, and G. Cipparrone, Attractive-repulsive dynamics on lightresponsive chiral microparticles induced by polarized tweezers, Lab. Chip 13, 459 (2013).

[21] G. Tkachenko and E. Brasselet, Spin Controlled Optical Radiation Pressure, Phys. Rev. Lett. 111, 033605 (2013).

[22] G. Tkachenko and E. Brasselet, Optofluidic chiral sorting of material chirality by chiral light, Nat. Commun. 5, 3577 (2014).

[23] G. Tkachenko and E. Brasselet, Helicity-dependent threedimensional optical trapping of chiral microparticles, Nat. Commun. 5, 4491 (2014).

[24] I. Dierking, Textures of Liquid Crystals (Wiley-VCH, Verlag GmbH \& Co. KGaA, Weinheim, 2003).

[25] Y. Bouligand and F. Livolant, The organization of cholesteric spherulites, J. Phys. 45, 1899 (1984).

[26] F. Xu and P. P. Crooker, Chiral nematic droplets with parallel surface anchoring, Phys. Rev. E 56, 6853 (1997).

[27] T. Orlova, T. Y. S. J. Asshoff, N. Katsonis, and E. Brasselet, Creation and manipulation of topological states in chiral nematic microspheres, Nat. Commun. 6, 7603 (2015).

[28] J.-K. Guo and J.-K. Song, Three-dimensional reconstruction of topological deformation in chiral nematic microspheres using fluorescence confocal polarizing microscopy, Opt. Express 24, 7381 (2016).

[29] G. Posnjak, S. Čopar, and I. Muševič, Points, skyrmions and torons in chiral nematic droplets, Sci. Rep. 6, 26361 (2016).

[30] J. Bezic and S. Zumer, Structures of the cholesteric liquid crystal droplets with parallel surface anchoring, Liq. Cryst. 11, 593 (1992).

[31] D. Sec, T. Porenta, M. Ravnik, and S. Zumer, Geometrical frustration of chiral ordering in cholesteric droplets, Soft Matter 8, 11982 (2012).

[32] G. E. Volovik and O. D. Lavrentovich, Topological dynamics of defects: boojums in nematic drops, Sov. Phys. JETP 58, 1159 (1983).

[33] M. Rafayelyan, G. Tkachenko, and E. Brasselet, Reflective SpinOrbit Geometric Phase from Chiral Anisotropic Optical Media, Phys. Rev. Lett. 116, 253902 (2016).

[34] J. Courtial, D. A. Robertson, K. Dholakia, L. Allen, and M. J. Padgett, Rotational Frequency Shift of a Light Beam, Phys. Rev. Lett. 81, 4828 (1998).

[35] S. Oka, Rheology (Academic Press, New York, 1960), Vol. 3.

[36] E. Brasselet, N. Murazawa, H. Misawa, and S. Juodkazis, Optical Vortices from Liquid Crystal Droplets, Phys. Rev. Lett. 103, 103903 (2009).

[37] M. Rafayelyan and E. Brasselet, Bragg-Berry mirrors: reflective broadband q-plates, Opt. Lett. 41, 3972 (2016).

[38] C. N. Alexeyev, Narrowband reflective generation of higherorder optical vortices in Bragg spun optical fibers, Appl. Opt. 52, 433 (2013). 